Pacific Journal of Mathematics

CERTAIN CONGRUENCES ON ORTHODOX SEMIGROUPS 


\title{
CERTAIN CONGRUENCES ON ORTHODOX SEMIGROUPS
}

\author{
Janet E. Mills
}

Letting $\kappa$ be the minimum unitary-congruence on a regular semigroup $S$ and $\xi$ be the minimum congruence such that $S / \xi$ is a semilattice of groups, it is the purpose of this paper to characterize all regular semigroups for which $\kappa \cap \xi$ is the identity relation. That is, we describe all regular semigroups which are subdirect products of a unitary semigroup and a semilattice of groups. In the process of doing this, a description of $\xi$ is given for any orthodox semigroup.

In A. H. Clifford's paper on radicals in semigroups, [2], a diagram was given presenting the relationship between various classes of regular semigroups and certain minimum congruences. Two questions were left open. The first was to find all subdirect products of a band and a semilattice of groups, that is, all semigroups for which $\beta \cap \xi$ is the identity, where $\beta$ is the minimum band-congruence. This was solved by Schein in [14] and also by Petrich in Theorem 3.2 of [11]. The second question involves finding all subdirect products of a semilattice of groups and a regular semigroup whose set of idempotents is unitary. In this paper we find that any such semigroup can be described as a semilattice of unitary semigroups on which $\mathscr{H} \cap \sigma$ is a unitary-congruence, where $\sigma$ is the minimum group-congruence. A description will also be given in terms of restrictions on the structure homomorphisms. In order to accomplish this, we first give an explicit characterization of $\xi$, the minimum semilattice of groups-congruence, on any orthodox semigroup.

1. Preliminary results.- For a regular semigroup $S, E_{S}$ denotes the set of idempotents of $S$. If $E_{s}$ is a subsemigroup then $S$ is said to be orthodox.

Proposition 1.1. [11; Proposition 2.5] On a regular semigroup $S$, the following are equivalent: for $s, t \in S$,

(i) $e$, es $\in E_{S}$ implies $s \in E_{S}$;

(ii) $e, s e \in E_{s}$ implies $s \in E_{s}$;

(iii) e, ese $\in E_{s}$ implies $s \in E_{s}$;

(iv) $e$, set $\in E_{S}$ implies st $\in E_{S}$;

(v) ese $=e \in E_{s}$ implies $s \in E_{s}$.

If any one of these five conditions holds, then $E_{S}$ is said to be unitary. For brevity, we shall call $S$ unitary if $E_{S}$ is a unitary subset of $S$. It is easily seen that any unitary semigroup is an orthodox semigroup. For inverse semigroups, those whose idempotents satisfy 
condition (v) are called proper by McAlister in [8] and [9], and he has given a description of all such inverse semigroups in terms of partially ordered sets and groups [9]. Unitary semigroups are not closed under homomorphisms; in fact, as is shown in [8], every inverse semigroup is an idempotent-separating homomorphic image of a unitary inverse semigroup.

A congruence on a semigroup is an equivalence relation which is compatible with multiplication. For two congruences $\rho, \rho^{\prime}$ on a semigroup $S, \rho \subseteq \rho^{\prime}$ if $a \rho b$ implies $a \rho^{\prime} b$. The identity relation on $S$ will be denoted by $\iota$, or $\iota_{S}$, if emphasis is needed. For the basic properties of congruences, the reader is referred to $[3 ; \S 1.4, \S 1.5]$.

For a class $\mathscr{C}$ of semigroups, a congruence $\rho$ is called a $\mathscr{C}$ congruence on $S$ if $S / \rho$ is in $\mathscr{C}$. For a regular semigroup, the following notation will be used:

$\kappa=$ the minimum unitary-congruence,

$\beta=$ the minimum band-congruence,

$\eta=$ the minimum semilattice-congruence,

$\mathscr{y}=$ the minimum inverse-congruence,

$\xi=$ the minimum semilattice of groups-congruence,

$\sigma=$ the minimum group-congruence,

$\mu=$ the maximum idempotent-separating congruence.

The Green relations will be noted as usual, and for brevity, a semilattice of groups-congruence will be called a $S G$-congruence. That each of the above minimum congruences exists is explained in [5], and also noted there are some of the following relationships which will be useful here:

$$
\mu \subseteq \mathscr{H} \subseteq \beta \subseteq \eta ; \quad \kappa \subseteq \beta \cap \sigma ; \quad \xi \subseteq \eta \cap \sigma .
$$

The following result will be needed for later work.

LEMMA 1.2. Let $\mathscr{B}$ be a class of regular semigroups and $\mathscr{C}$ be a subclass of $\mathscr{B}$ such that for any $S \in \mathscr{B}$, the minimum $\mathscr{C}$-congruence on $S, \rho$, exists. If $\tau$ is any congruence defined on all semigroups in $\mathscr{B}$ such that $\tau$ is the identity on any $\mathscr{C}$-semigroup, then $\tau \subseteq \rho$ in $\mathscr{B}$.

Proof. Let $\tau$ be such a congruence, $S \in \mathscr{B}$. Then $\tau \vee \rho$ is a congruence and $\tau \vee \rho=\iota$ on any $\mathscr{C}$-semigroup. Now $S / \rho$ is a $\mathscr{C}$ semigroup and $\rho \subseteq \tau \vee \rho$, so $(S / \rho) /(\tau \vee \rho) / \rho=S / \rho / \iota=S / \rho$. On the other hand, $(S / \rho) /(\tau \vee \rho) / \rho=S /(\tau \vee \rho)$. Hence $S / \rho \simeq S /(\tau \vee \rho)$. Therefore $\rho=$ $\tau \vee \rho$ and $\tau \subseteq \rho$.

Let $\left\{S_{\alpha}\right\}_{\alpha \in A}$ be a family of semigroups and $T$ be a subsemigroup of the direct product $\prod_{\alpha \in A} S_{\alpha}$. For each $\alpha \in A, \pi_{\alpha}$ is the natural projection of $T$ into $S_{\alpha}$. A semigroup $S$ is a subdirect product of $S_{\alpha}, \alpha \in A$, if $S$ is 
isomorphic to a subsemigroup $T$ of $\prod_{\alpha \in A} S_{\alpha}$ such that $T \pi_{\alpha}=S_{\alpha}$ for all $\alpha$ in $A$. For the particular case we are interested in, the relationship between congruences and subdirect products is as follows (see [10; II.1.4]). For congruences $\lambda, \rho$ on a semigroup $S, S$ is a subdirect product of $S / \lambda$ and $S / \rho$ if and only if $\lambda \cap \rho=\iota_{s}$. The aim of this paper is to describe all subdirect products of a unitary semigroup and a semilattice of groups. It is evident that this is equivalent to finding all semigroups for which $\kappa \cap \xi$ is the identity congruence. It is the latter attack that we shall make. From now on, we will assume that all semigroups are regular.

2. The minimum semilattice of groups-congruence. It was shown in [5] that $\eta \cap \sigma$ is the smallest congruence $\rho$ such that $S / \rho$ is a semilattice of groups and is unitary. Thus, in general, $\xi$ is strictly contained in $\eta \cap \sigma$. Recall [7; Theorem 3.1] that on an orthodox semigroup $S$,

$$
a \sigma b \leftrightarrow e a e=e b e \text { for some } e \text { in } E_{s} .
$$

To find $\xi$ on any orthodox semigroup, we first describe $\xi$ on any inverse semigroup and extend it to an orthodox semigroup via the method developed in Theorem 3.1 of [7].

THEOREM 2.1. Let $S$ be an inverse semigroup. The minimum SGcongruence $\xi$ on $S$ can be defined as follows:

$$
a \xi b \leftrightarrow a \eta b \text { and } e a=e b \text { for some } e^{2}=e \eta a .
$$

Proof. Let $a \tau b$ if and only if $a \eta b$ and $e a=e b$ for some $e^{2}=e \eta a$. It is easily seen that $\tau$ is an equivalence relation on $S$. Let $a \tau b$ and $x$ be in $S$. Then $a \eta b$ and $e a=e b$ for some $e^{2}=e \eta a$. Since $\eta$ is a congruence, $a x \eta b x$. Let $f$ be any idempotent such that $f \eta x$. Then ef $\eta a x$ and

$$
(e f)(a x)=f(e a) x=f(e b) x=(f e)(b x)=(e f)(b x) \text {; }
$$

therefore, $a x \tau b x$. On the other hand, $x a \eta x b$ and $x e \eta x a$. Thus, since $\eta$ is a semilattice congruence, $x e x^{-1} \eta x a$. In addition,

$$
\left(x e x^{-1}\right)(x a)=x e\left(x^{-1} x\right) a=x e a=x e b=\left(x e x^{-1}\right)(x b) \text {; }
$$

that is, $x a \tau x b$.

To see that $S / \tau$ is a semilattice of groups, it is sufficient to show that $a a^{-1} \tau a^{-1} a$ for all $a$ in $S$. But this is clear by letting $e=\left(a a^{-1}\right)\left(a^{-1} a\right)$. Therefore, $\xi \subseteq \tau$.

Now if $S$ is in fact a semilattice of groups then $\eta=\mathscr{H}$ and $\tau$ is clearly the identity on $S$. Hence by Lemma 1.2, $\tau \subseteq \xi$. Consequently $\tau=\xi$. 
On an orthodox semigroup, the minimum inverse-congruence $y$ has been described by Hall [4] and Schein [13] as follows:

$$
a \mathscr{Y} b \leftrightarrow V(a)=V(b),
$$

where $V(x)$ is the set of all inverses of $x$. For any $a, a y$ will denote the $y$-class containing $a$.

THEOREM 2.2. Let $S$ be an orthodox semigroup. Then $\xi$ can be defined on $S$ as follows:

$$
a \xi b \leftrightarrow a \eta b \text { and } e a e=e b e \text { for some } e^{2}=e \eta a .
$$

Proof. Since $\mathscr{Y}$ is the minimum inverse-congruence then $\mathscr{Y} \subseteq$ $\eta$. Thus for $a, b \in S, a \mathscr{Y}_{\eta} b \mathscr{Y}$ implies $a \eta b$.

Now, $S / \mathscr{Y}$ is the maximum inverse homomorphic image of $S$, and therefore, letting $\xi^{\prime}$ be the minimum $S G$-congruence on $S / Y$, we have, via Theorem 2.1,

$$
a \xi b \leftrightarrow a \mathscr{Y} \xi^{\prime} b \mathscr{Y} \leftrightarrow a \mathscr{Y} \eta b^{\mathscr{Y}} \quad \text { and } \quad x \mathscr{Y} a \mathscr{Y}=x \mathscr{Y} b \mathscr{Y} \text {, }
$$

for some $(x \mathscr{Y})^{2}=x \mathscr{Y}$ with $x \mathscr{Y} \eta a \mathscr{Y}$.

Since $(x \mathscr{Y})^{2}=x \mathscr{Y}$, there exists an idempotent $f$ such that $f \mathscr{Y} x$, and thus $f \eta a$. Therefore, using the fact that $\mathscr{Y}$ is a congruence, $(*)$ is equivalent to

$$
a \mathscr{Y}_{\eta} b \mathscr{Y} \text { and }(f a)^{\mathscr{Y}}=(f b)^{\mathscr{Y}} \text { for some } f^{2}=f \eta a \text {. }
$$

By definition of $\mathscr{Y}$, this means

$$
a \xi b \leftrightarrow a \eta b \quad \text { and } \quad V(f a)=V(f b) \text { for some } f^{2}=f \eta a .
$$

The rest of the proof that $\xi$ can be defined as in the statement of the theorem is very similar to that of Lemma 3.2 of [7], using the additional fact that $\eta$ is a semilattice-congruence.

COROllary 2.3. (See [14] or [11; Theorem 3.2].) Let $S$ be a regular semigroup. Then $\beta \cap \xi=\iota_{S}$ if and only if $S$ is an orthodox band of groups.

Proof. Let $\beta \cap \xi=\iota_{s}$. Now we know that $\mathscr{H} \subseteq \beta \subseteq \eta$. We will show that $\beta$ is idempotent-separating. Let $e$ and $f$ be idempotents with $e \beta f$. Then enf and $(e f) e(e f)=(e f) f(e f)$ with $e f \eta e$. That is, by Theorem 2.2, $e \xi f$. Since $\beta \cap \xi=\iota$, we have $e=f$. Therefore, $\beta \subseteq \mu \subseteq \mathscr{H}$. 
But $\mathscr{H} \subseteq \beta$ so $\beta=\mathscr{H}$ and $S$ is a band of groups. The converse follows easily from the fact that $\beta=\mathscr{H}$ and $\mathscr{H} \cap \xi=\iota$.

3. $\kappa \cap \xi=\iota$. In this section we characterize those semigroups $S$ which are a subdirect product of a unitary semigroup and a semilattice of groups, that is, those semigroups $S$ for which $\kappa \cap \xi$ is the identity. Clearly, since a unitary semigroup and a semilattice of groups are both orthodox, then $S$ is again an orthodox semigroup.

Recall that a semigroup is $\eta$-simple if it has exactly one $\eta$-class.

Lemma 3.1. Let $S$ be an $\eta$-simple orthodox semigroup. Then $S$ is unitary if and only if $\kappa \cap \xi=\iota_{s}$.

Proof. If $S$ is unitary then $\kappa=\iota_{S}$ so $\kappa \cap \xi=\iota_{s}$. Conversely, let $\kappa \cap \xi=\iota_{s}$. Then, using Theorem 2.2 and the fact that $S$ is $\eta$-simple, we have

$$
\begin{gathered}
a \xi b \leftrightarrow a \eta b \text { and } e a e=e b e \text { for some } e^{2}=e \eta a \\
\leftrightarrow e a e=e b e \text { for some } e^{2}=e \leftrightarrow a \sigma b .
\end{gathered}
$$

That is, $\xi=\sigma$ and $\kappa \cap \sigma=\iota_{s}$. But $\sigma$ is a unitary congruence so $\kappa \cap \sigma=\kappa$. Therefore $\kappa=\iota_{S}$ and $S$ is unitary.

Lemma 3.2. Let $S$ be a semigroup with $\kappa \cap \xi=\iota_{s}$. Then $S$ is a semilattice of $\eta$-simple unitary semigroups.

Proof. Since $\eta$ is a semilattice-congruence, we know that $S$ is a semilattice $Y$ of $\eta$-simple semigroups $S_{\alpha}, \alpha \in Y$. Now, on $S_{\alpha}, \kappa \mid S_{\alpha}$ is a unitary-congruence and $\xi \mid S_{\alpha}$ is a $S G$-congruence. Hence, on $S_{\alpha}$, $\left(\kappa \mid S_{\alpha}\right) \cap\left(\xi \mid S_{\alpha}\right)=\iota$, and thus the intersection of the minimum unitarycongruence on $S_{\alpha}$ and the minimum $S G$-congruence on $S_{\alpha}$ is also the identity. By Lemma 3.1, $S_{\alpha}$ is unitary.

LEMMA 3.3 [5; Theorem 3.9]. If $S$ is a unitary semigroup then $\mathscr{H} \cap \sigma=\iota_{s}$.

Lemma 3.4. Let $S$ be a regular semigroup. Then $\mathscr{H} \cap \sigma \subseteq \kappa$.

Proof. Let $\mathscr{K}$ be the class of all unitary semigroups. Letting $\tau$ be the congruence generated by $\mathscr{H} \cap \sigma$, then $\tau=\iota_{s}$ for any $S \in \mathscr{K}$, by Lemma 3.3. Therefore, by Lemma $1.2, \tau \subseteq \kappa$. That is, $\mathscr{H} \cap \sigma \subseteq \kappa$.

THEOREM 3.5. Let $S$ be a regular semigroup. The following statements are equivalent. 
(i) $\kappa \cap \xi=\iota_{s}$, where $\kappa$ is the minimum unitary-congruence and $\xi$ is the minimum $S G$-congruence.

(ii) $S$ is a semilattice of unitary semigroups and $\kappa=\mathscr{H} \cap \sigma=$ $\mu \cap \sigma$.

(iii) $S$ is a semilattice of unitary semigroups and $\mathscr{H} \cap \sigma$ is a unitary congruence on $S$.

(iv) $S$ is a subdirect product of a unitary semigroup and a semilattice of groups.

Proof. (i) implies (ii). Let $\kappa \cap \xi=\imath$. By Lemma 3.2, S is a semilattice of unitary semigroups. Since $\eta$ and $\sigma$ are both unitarycongruences, $\kappa$ is contained in both $\eta$ and $\sigma$. We shall show that $\kappa$ is idempotent-separating. For, let $e \kappa f$, with $e, f \in E_{s}$. Then enf and $(e f) e(e f)=(e f) f(e f)$ with efne. That is, by Theorem $2.2, e \xi f$. Since $\kappa \cap \xi=\imath$, then $e=f$. Hence $\kappa$ is idempotent-separating and $\kappa \subseteq \mu$. Consequently, using Lemma 3.4, we have $\mathscr{H} \cap \sigma \subseteq \kappa \subseteq \mu \cap \sigma$. But $\mu \subseteq \mathscr{H}$, so equality holds.

(ii) implies (iii). Clear.

(iii) implies (iv). Let $S$ be a semilattice of $\eta$-simple unitary semigroups $S_{\alpha}, \alpha \in Y$, with $\mathscr{H} \cap \sigma$ unitary. Then by Lemma $3.4, \kappa=$ $\mathscr{H} \cap \sigma$. Therefore

$$
\kappa \cap \xi=(\mathscr{H} \cap \sigma) \cap \xi=\mathscr{H} \cap(\sigma \cap \xi)=\mathscr{H} \cap \xi
$$

Let $a \mathscr{H} \cap \xi b$. Then $a, b \in S_{\alpha}$ for some $\alpha$. Thus, since $S_{\alpha}$ is $\eta$-simple, in $S_{\alpha}, a \mathscr{H} \cap \sigma b$. But $S_{\alpha}$ is unitary, so by Lemma 3.3, on $S_{\alpha}, \mathscr{H} \cap \sigma=\imath$. Hence $a=b$. Consequently $\kappa \cap \xi=\iota_{s}$. Therefore $S$ is a subdirect product of $S / \kappa$ and $S / \xi$ (see II.1.4 of [10]).

(iv) implies (i). Let $S$ be a subdirect product of a unitary semigroup $U$ and a semilattice of groups $T$. Then the congruences induced on $S$ by the two projection maps are, respectively, a unitary-congruence $\lambda$, and a $S G$-congruence, $\rho$, and $\lambda \cap \rho=\iota_{s}$. Thus $\kappa \cap \xi \subseteq \lambda \cap \rho=\iota_{s}$.

It is not possible to eliminate either one of the two conditions:

(1) $S$ is a semilattice of unitary semigroups,

(2) $\mathscr{H} \cap \sigma$ is unitary.

For, any unitary semigroup (which is not a group) with a zero adjoined, satisfies (1), but for such a semigroup, $\kappa=\beta$ and $\beta \cap \xi \neq \iota$ by Corollary 2.3. On the other hand, let $S=B(G, \alpha)$ be any bisimple $\omega$-semigroup for which $\alpha$ is not one-to-one. Then $S$ is not unitary but $\kappa=\mathscr{H} \cap \sigma$ and $\kappa \cap \xi=\kappa \cap \sigma=\mathscr{H} \cap \sigma \neq \iota$.

COROllary 3.6. Let $S$ be a fundamental regular semigroup. Then $\kappa \cap \xi=\iota$ if and only if $S$ is unitary. 
Proof. Since $S$ is fundamental, then $\mu=\iota$. Therefore, $\mu \cap \sigma=\imath$.

Every regular semigroup which is a semilattice $Y$ of semigroups $S_{\alpha}$, $\alpha \in Y$, can be constructed via certain homomorphisms $\phi_{\alpha, \beta}$ from $S_{\alpha}$ into $\Omega\left(S_{\beta}\right)$, the translational hull of $S_{\beta}$, for all $\alpha>\beta$; in this case, we shall denote $S$ by $\left(Y, S_{\alpha}, \phi_{\alpha, \beta}\right)$. For a full description of this structure the reader is referred to [10; III.7.5]. In light of Theorem 3.5, it is of interest to know how the condition $\kappa=\mathscr{H} \cap \sigma$ can be expressed in terms of the structure homomorphisms $\phi_{\alpha, \beta}$. To do this we need to explore the translational hull $\Omega(T)$ of a unitary semigroup $T$. For the elementary properties of the translational hull, see Chapter V of [10]. Recall [10; III.7.5] that in $S=\left(Y, S_{\alpha}, \phi_{\alpha, \beta}\right)$, if $s \in S_{\alpha}, t \in S_{\beta}$, with $\alpha>\beta$ then

$$
s t=\phi_{\alpha, \beta}^{s} t=\lambda^{s} t \quad \text { and } \quad t s=t \phi_{\alpha, \beta}^{s}=t \rho^{s},
$$

where $\phi_{\alpha, \beta}^{s}=\left(\lambda^{s}, \rho^{s}\right) \in \Omega\left(S_{\beta}\right)$.

LEMMA 3.7. Let $S$ be unitary and $(\lambda, \rho) \in \Omega(S)$. If there exists an idempotent e such that $\lambda e \in E_{S}$ or $e \rho \in E_{s}$ then $\lambda\left(E_{S}\right) \subseteq E_{S},\left(E_{S}\right) \rho \subseteq E_{s}$.

Proof. Let $e$ and $\lambda e$ be in $E_{s}$. Then $(e \rho) e=e(\lambda e) \in E_{S}$, and since $S$ is unitary, by Proposition $1.1, e \rho \in E_{s}$.

Let $f$ be in $E_{s}$. Then $e(\lambda f)=(e \rho) f \in E_{s}$, so again $\lambda f$ is in $E_{s}$. Thus $\lambda\left(E_{S}\right) \subseteq E_{s}$. Since $\lambda f \in E_{s}$ implies $f \rho \in E_{s}$, then also $\left(E_{S}\right) \rho \subseteq E_{S}$.

Lemma 3.8. Let $S$ be a unitary semigroup. Define

$$
K(S)=\left\{(\lambda, \rho) \in \Omega(S) \mid \lambda\left(E_{S}\right) \subseteq E_{S},\left(E_{S}\right) \rho \subseteq E_{S}\right\}
$$

Then $K(S)$ is a subsemigroup of $\Omega(S)$ which contains $E_{\Omega(S)}$.

Proof. That $K(S)$ is a semigroup is clear. Let $(\lambda, \rho)$ be in $E_{\Omega(S)}$. Then $\lambda^{2}=\lambda, \rho^{2}=\rho$. Let $a \in S$ and $\lambda a=b$. Let $b^{\prime}$ be an inverse of $b$; then we have $\lambda\left(a b^{\prime}\right)=(\lambda a) b^{\prime}=b b^{\prime} \in E_{s}$. Hence there exists $x$ in $S$ such that $\lambda x=f \in E_{s}$. Moreover, $f=\lambda x=\lambda^{2} x=\lambda(\lambda x)=\lambda f$. Therefore $\lambda f \in E_{S}$, and by Lemma 3.7, $\lambda\left(E_{S}\right) \subseteq E_{s}$. Similarly $\left(E_{S}\right) \rho \subseteq E_{s}$.

If $S$ is an inverse semigroup then $E_{\Omega(S)}=K(S),[\mathbf{1}$; Lemma 2.1]. However, in general, strict containment is possible. For, if $S$ is a rectangular group, we may assume $S=L \times G \times R$ where $L(R)$ is. a left (right) zero semigroup and $G$ is a group. Then $\Omega(S)=$ $T(L) \times G \times T^{\prime}(R)$, where $T(L)\left(T^{\prime}(R)\right)$ is the semigroup of all transformations of $L(R)$ written on the left (right) [10; V.3.12]. Under this isomorphism, 


$$
E_{\Omega(s)}=\left\{\left(f, 1, f^{\prime}\right) \mid f, f^{\prime} \text { are retractions }\right\},
$$

where a retraction is any mapping which is the identiy on its range. On the other hand, $K(S)=T(L) \times 1 \times T^{\prime}(R)$ which is not equal to $E_{\Omega(S)}$.

From [5] we recall that a congruence $\tau$ is unitary if $x^{2} \tau x$ and $(s x)^{2} \tau s x$ implies $s^{2} \tau s$. For regular semigroups this is equivalent to:

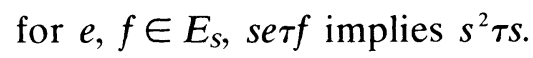

We now explore the properties of $\phi_{\alpha, \beta}$ which make $\kappa \cap \xi$ the identity. For a semigroup $S_{\alpha}$, we denote $E_{S_{\alpha}}$ by $E_{\alpha}$, and $K\left(S_{\alpha}\right)$ by $\dot{K_{\alpha}}$. For $S=\left(Y, S_{\alpha}, \phi_{\alpha, \beta}\right)$ a semilattice of unitary semigroups $S_{\alpha}$, let $\Gamma_{\alpha, \beta}=K_{\beta} \phi_{\alpha, \beta}^{-1}$ for all $\alpha>\beta$ and $\Gamma=\bigcup_{\alpha>\beta} \Gamma_{\alpha, \beta}$.

THEOREM 3.9. Let $S$ be a regular semigroup. Then $\kappa \cap \xi=\iota_{S}$ if and only if $S=\left(Y, S_{\alpha}, \phi_{\alpha, \beta}\right)$ is a semilattice of unitary semigroups satisfying the properties:

(i) $\Gamma$ is a band of groups,

(ii) for $s$ in $\Gamma \cap H_{e}, e \in E_{\alpha}$, if $f<e$ with $f \in E_{\beta}$ then $\phi_{\alpha, \beta}^{s}(f) \mathscr{H} f$, where $\phi_{\alpha, \beta}^{s}(f)$ means both $\phi_{\alpha, \beta}^{s} f$ and $f \phi_{\alpha, \beta}^{s}$.

Proof. Let $\kappa \cap \xi=\iota_{s}$. Then $\mathscr{H} \cap \sigma=\mu \cap \sigma$ is unitary. Let $\alpha>\beta$ and $s$ be in $S_{\alpha}$ with $\phi_{\alpha, \beta}^{s} \in K_{\beta}$. Then $\phi_{\alpha, \beta}^{s}=(\lambda, \rho)$ and $\lambda\left(E_{\beta}\right) \subseteq E_{\beta}$. Let $g$ be in $E_{\beta}$. Then $\lambda g=f=f^{2} \in E_{\beta}$, so by definition of multiplication in $S$, $s g=\phi_{\alpha, \beta}^{s} g=\lambda g=f$. Hence se $\mu \cap \sigma f$, and $\mu \cap \sigma$ is unitary, so $s \mu \cap \sigma s^{2}$. Thus $s$ is contained in a group. Now since $\mu \cap \sigma$ is a congruence, the $\mu \cap \sigma$-classes which contain idempotents form a band of groups, $T$, and since $\phi_{\alpha, \beta}$ is a homomorphism, then $K_{\beta} \phi_{\alpha, \beta}^{-1}=\Gamma_{\alpha, \beta}$ is a band of groups contained in $T$. Thus $\Gamma$ is a band of groups.

Now let $s$ be in $\Gamma_{\alpha, \beta}$. Then $s$ is in a group so there exists an idempotent $h$ such that $s s^{\prime}=s^{\prime} s=h$ for some $s^{\prime} \in V(s)$. Since $s \mu s^{2}$, then $s \mu h$ and for all idempotents $f, s f s^{\prime}=h f h, s^{\prime} f s=h f h,[6]$.

Let $f<h, f \in S_{\gamma}$. Then $s f s^{\prime}=f$ and $f s^{\prime} s f=f h f=f$. Thus if $\phi_{\alpha, \gamma}^{s}=$ $\left(\lambda^{s}, \rho^{s}\right)$ and $\phi_{\alpha, \gamma}^{s}=\left(\lambda^{s^{\prime}}, \rho^{s^{\prime}}\right), f=f s^{\prime} s f=\left(f \rho^{s^{\prime}}\right)\left(\lambda^{s} f\right)$. Therefore,

$$
f \rho^{s^{\prime}}=f\left(f \rho^{s^{\prime}}\right)=\left(f \rho^{s^{\prime}}\right)\left(\lambda^{s} f\right)\left(f \rho^{s^{\prime}}\right), \quad \lambda^{s} f=\left(\lambda^{s} f\right) f=\left(\lambda^{s} f\right)\left(f \rho^{s^{\prime}}\right)\left(\lambda^{s} f\right) ;
$$

that is, $f \rho^{s^{\prime}}$ is an inverse of $\lambda^{s} f$. Now $s f s^{\prime}=f=f s^{\prime} s f$ can be expressed by

$$
\left(\lambda^{s} f\right)\left(f \rho^{s^{\prime}}\right)=f=\left(f \rho^{s^{\prime}}\right)\left(\lambda^{s} f\right)
$$

Thus $\lambda^{s} f \mathscr{H} f$. By considering $s^{\prime} f s=f s s^{\prime} f=f$ we find $f \rho^{s} \mathscr{H} f$. Thus $\phi_{\alpha, \gamma}^{s}(f) \mathscr{H} f$.

Conversely, to show $\kappa \cap \xi=\iota_{s}$, using Theorem 3.5, we need only show that $\mu \cap \sigma$ is a unitary congruence. Let $\operatorname{se} \mu \cap \sigma f$ with $e, f \in E_{s}$. 
Letting $s$ be in $S_{\alpha}, e$ in $E_{\beta}$, then $f \in E_{a \beta}$. Since $\operatorname{se} \sigma f$, there exists $g \in E_{\gamma}$, $\gamma \leqq \alpha \beta$ such that $g(s e) g=g f g \in E_{\gamma}$. This means that $\left(g \phi_{\alpha, \gamma}^{s}\right)\left(\phi_{\beta, \gamma}^{e} g\right) \in E_{\gamma}$. Since $e$ is idempotent so is $\phi_{\beta, \gamma}^{e}$ and by Lemma 3.8, $\phi_{\beta, \gamma}^{e} g$ is in $E_{\gamma}$. Thus since $S_{\gamma}$ is unitary, $g \phi_{\alpha, \gamma}^{s}$ is idempotent by Proposition 1.1; by Lemma 3.7, $\phi_{\alpha, \gamma}^{s}$ is in $K_{\gamma}$. Consequently by property (i), $s$ is contained in a band $E_{\alpha}$ of groups $G_{b}, b \in E_{\alpha}$. In particular, there exists $s^{\prime} \in V(s)$ such that $s s^{\prime}=$ $s^{\prime} s=h$ for some $h \in E_{\alpha}$. We need to show that $s \mu h$, and to do this it is sufficient to show that $s f s^{\prime}=f, s^{\prime} f s=f$ for all $f \leqq h$. Now if $f$ is in $E_{\alpha}$, $f \leqq h$, then $s$ and $s^{\prime}$ are in the group $G_{h}$ and

$$
s f s^{\prime} \in G_{h} G_{f} G_{h} \subseteq G_{h f h}=G_{f},
$$

so that $s f s^{\prime}=f$. Similarly $s^{\prime} f s=f$. Now let $f$ be in $E_{\delta}, \delta<\alpha$, with $f<h$. Let $\phi_{\alpha, \delta}^{s}=\left(\lambda^{s}, \rho^{s}\right), \phi_{\alpha, \delta}^{s^{\prime}}=\left(\lambda^{s^{\prime}}, \rho^{s^{\prime}}\right)$. By property (ii), $\lambda^{s} f \mathscr{H} f, f \rho^{s} \mathscr{H} f$. That is, $f\left(\lambda^{s} f\right)=\lambda^{s} f,\left(f \rho^{s}\right) f=f \rho^{s}$. Now since $f<h=s s^{\prime}$, then $f=h f=$ $\lambda^{s s^{\prime}} f=\lambda^{s} \lambda^{s^{\prime}} f$, and thus

$$
\begin{aligned}
s f s^{\prime} & =\left(\lambda^{s} f\right)\left(f \rho^{s^{\prime}}\right)=f\left(\lambda^{s} f\right)\left(f \rho^{s^{\prime}}\right)=\left(f \rho^{s}\right) f\left(f \rho^{s^{\prime}}\right)=\left(f \rho^{s}\right)\left(f \rho^{s^{\prime}}\right) \\
& =\left[\left(f \rho^{s}\right) f\right] \rho^{s^{\prime}}=\left(f \rho^{s}\right) \rho^{s^{\prime}}=f \rho^{s s^{\prime}}=f .
\end{aligned}
$$

Similarly $s^{\prime} f s=f$. Therefore $s \mu h$.

Since $\sigma$ is always unitary, seof implies soh. Consequently, $s \mu \cap \sigma h$, and $\mu \cap \sigma$ is a unitary congruence. By Theorem 3.5, $\kappa \cap \xi=\iota_{s}$.

A regular semigroup $S=\left(Y, S_{\alpha}, \phi_{\alpha, \beta}\right)$ is a strong semilattice of the semigroups $S_{\alpha}$, if $\phi_{\alpha, \beta}$ maps $S_{\alpha}$ into $S_{\beta}$ for all $\alpha>\beta$. The conditions in Theorem 3.9 can be simplified considerably for strong semilattices of unitary semigroups.

Corollary 3.10. Let $S=\left(Y, S_{\alpha}, \phi_{\alpha, \beta}\right)$ be a strong semilattice of unitary semigroups $S_{\alpha}$. Then $\kappa \cap \xi=\iota_{s}$ if and only if $E_{\beta} \phi_{\alpha, \beta}^{-1}$ is a band of groups for all $\alpha>\beta$.

Proof. It can be easily seen that $K_{\beta} \cap \Pi\left(S_{\beta}\right) \simeq E_{\beta}$ where $\Pi\left(S_{\beta}\right)$ is the semigroup of inner bitranslations of $S_{\beta}$. Thus if $\phi_{\alpha, \beta}$ maps $S_{\alpha}$ into $S_{\beta} \simeq \Pi\left(S_{\beta}\right)$, then $K_{\beta} \phi_{\alpha, \beta}^{-1}=E_{\beta} \phi_{\alpha, \beta}^{-1}$. Property (ii) automatically holds since homomorphisms preserve $\mathscr{H}$-classes.

The author would like to express her appreciation to Professor Mario Petrich for the suggestions he made on the preliminary copy of this paper.

\section{REFERENCES}

1. Janet E. Ault, The translational hull of an inverse semigroup, Glasgow Math. J., 14 (1973), 56-64.

2. A. H. Clifford, Radicals in semigroups, Semigroup Forum, 1 (1970), 103-128. 
3. A. H. Clifford and G. B. Preston, The algebraic theory of semigroups, Amer. Math. Soc., Math. Surveys No. 7, Vol. I, Providence, R.I., 1961.

4. T. E. Hall, On regular semigroups whose idempotents form a subsemigroup, Bull. Austral. Math. Soc., 1 (1969), 195-208.

5. J. M. Howie and G. Lallement, Certain fundamental congruences on a regular semigroup, Proc. Glasgow Math. Assoc., 7 (1966), 145-159.

6. John Meakin, Congruences on orthodox semigroups, J. Austral. Math. Soc., 12 (1971), 323-341.

7. _ Congruences on orthodox semigroups II, J. Austral. Math. Soc., 13 (1972), 259-266.

8. D. B. McAlister, Groups, semilattices and inverse semigroups, Trans. Amer. Math. Soc., 192 (1974), 227-244.

9. ——, Groups, semilattices and inverse semigroups, II, Trans. Amer. Math. Soc., 196 (1974), 351-370.

10. Mario Petrich, Introduction to Semigroups, Charles E. Merrill Publishing Co., Columbus, Ohio, 1973.

11. - Regular semigroups which are subdirect products of a band and a semilattice of groups, Glasgow Math. J., 14 (1973), 27-49.

12. N. R. Reilly and H. E. Scheiblich, Congruences on regular semigroups, Pacific J. Math., 23 (1967), 349-360.

13. B. M. Schein, On the theory of generalized groups and generalized heaps, Theory of semigroups and appl. I, 286-324, Izdat. Saratov. Univ., Saratov, 1965 (Russian).

14. — A note on radicals in regular semigroups, Semigroup Forum, 3 (1971), 84-85.

Received September 29, 1975.

Madison College 



\section{Pacific Journal of Mathematics}

Vol. 64, No. 1

May, 1976

Walter Allegretto, Nonoscillation theory of elliptic equations of order $2 n \ldots \ldots \quad 1$

Bruce Allem Anderson, Sequencings and starters.................. 17

Friedrich-Wilhelm Bauer, A shape theory with singular homology .......... 25

John Kelly Beem, Characterizing Finsler spaces which are

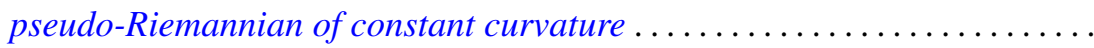

Dennis K. Burke and Ernest A. Michael, On certain point-countable

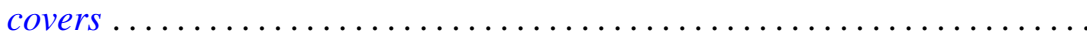

Robert Chen, A generalization of a theorem of Chacon ............... 93

Francis H. Clarke, On the inverse function theorem ................ 97

James Bryan Collier, The dual of a space with the Radon-Nikodým

property ....................................... 103

John E. Cruthirds, Infinite Galois theory for commutative rings ............ 107

Artatrana Dash, Joint essential spectra......................... 119

Robert M. DeVos, Subsequences and rearrangements of sequences in FK

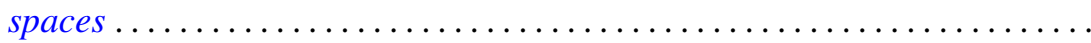

Geoffrey Fox and Pedro Morales, Non-Hausdorff multifunction generalization

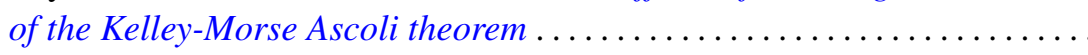

Richard Joseph Fleming, Jerome A. Goldstein and James E. Jamison, One

parameter groups of isometries on certain Banach spaces.............

Robert David Gulliver, II, Finiteness of the ramified set for branched

immersions of surfaces

Kenneth Hardy and István Juhász, Normality and the weak cb property ...... 167

C. A. Hayes, Derivation of the integrals of $L^{(q)}$-functions.

Frederic Timothy Howard, Roots of the Euler polynomials .

Robert Edward Jamison, II, Richard O'Brien and Peter Drummond Taylor, On

embedding a compact convex set into a locally convex topological vector space ....................................

Andrew Lelek, An example of a simple triod with surjective span smaller than span ...

Janet E. Mills, Certain congruences on orthodox semigroups

Donald J. Newman and A. R. Reddy, Rational approximation of $e^{-x}$ on the positive real axis.

John Robert Quine, Jr., Homotopies and intersection sequences ...

Nambury Sitarama Raju, Periodic Jacobi-Perron algorithms and fundamental units ....

Herbert Silverman, Convexity theorems for subclasses of univalent functions. . .

Charles Frederick Wells, Centralizers of transitive semigroup actions and endomorphisms of trees.........................

Volker Wrobel, Spectral approximation theorems in locally convex spaces ..................... 